This item was submitted to Loughborough's Research Repository by the author.

Items in Figshare are protected by copyright, with all rights reserved, unless otherwise indicated.

\title{
Effect of Al substitution on the magnetocaloric properties of La(Fe SiAl)
}

PLEASE CITE THE PUBLISHED VERSION

http://dx.doi.org/10.1103/PhysRevB.83.144415

\section{PUBLISHER}

(C) American Physical Society

\section{VERSION}

VoR (Version of Record)

\section{LICENCE}

CC BY-NC-ND 4.0

\section{REPOSITORY RECORD}

Morrison, Kelly, S.M. Podgornykh, Y.V. Shcherbakova, A.D. Caplin, and L.F. Cohen. 2019. "Effect of Al Substitution on the Magnetocaloric Properties of La(fe Sial)". figshare. https://hdl.handle.net/2134/12672. 
This item was submitted to Loughborough's Institutional Repository (https://dspace.lboro.ac.uk/) by the author and is made available under the following Creative Commons Licence conditions.

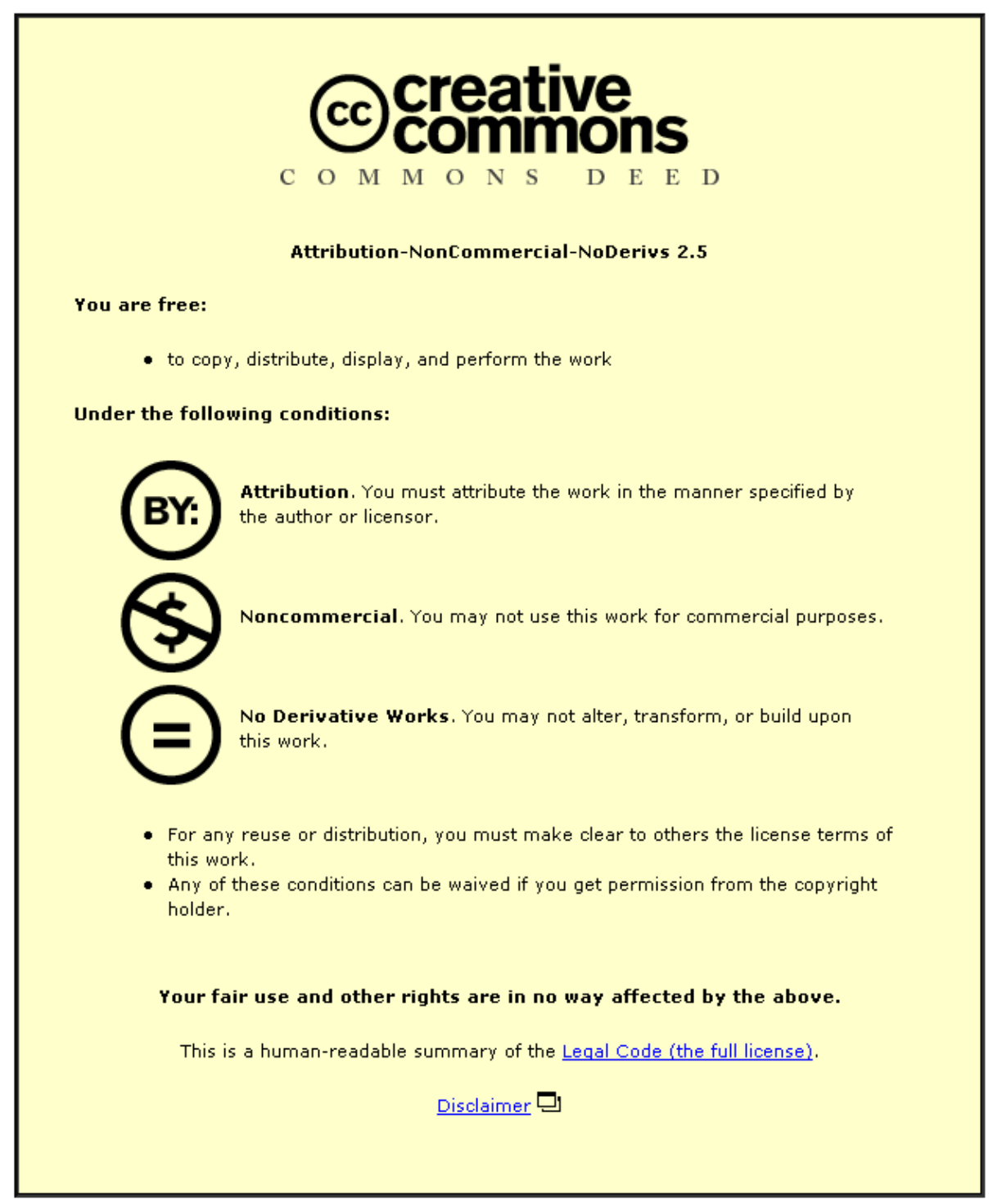

For the full text of this licence, please go to: http://creativecommons.org/licenses/by-nc-nd/2.5/ 


\title{
Effect of Al substitution on the magnetocaloric properties of $\mathrm{La}\left(\mathrm{Fe}_{0.88} \mathrm{Si}_{0.12-x} \mathrm{Al}_{x}\right)_{13}$
}

\author{
K. Morrison, ${ }^{1}$ S.M. Podgornykh, ${ }^{2}$ Ye.V. Shcherbakova, ${ }^{2}$ A.D. Caplin,,${ }^{1}$ and L.F. Cohen ${ }^{1}$ \\ ${ }^{1}$ The Blackett Laboratory, Imperial College, London SW7 2BZ, UK \\ ${ }^{2}$ Institute of Metal Physics, Ural Division of the Russian Academy of Sciences, S. Kovalevskya Street, 18, Ekaterinburg 620219 (Russia)
}

(Received 17 October 2010; revised manuscript received 23 December 2010; published 19 April 2011)

\begin{abstract}
Here we study the influence of $\mathrm{Al}$ doping on the magnetization, heat capacity, and entropy change of $\mathrm{La}\left(\mathrm{Fe}_{0.88} \mathrm{Si}_{0.12-x} \mathrm{Al}_{x}\right)_{13}$ where $x=0,0.048$, and 0.081 . When $x=0$, the system shows a remarkably sharp heat capacity feature associated with spin fluctuations coincident with, but quite distinct from the latent heat spike of the first order paramagnetic to ferromagnetic phase transition. With the addition of Al the magnetic and calorimetric features become more distributed in field, suggesting that $\mathrm{Al}$ adds disorder to the system. For both finite $x$ compositions studied here, the latent heat disappears and the transition can be classified as second order. Although the entropy change associated with the transition is reduced once $\mathrm{Al}$ is substituted for $\mathrm{Si}$, the adiabatic temperature change, $\Delta T_{\text {ad }}$ is still significant. In $\mathrm{La}\left(\mathrm{Fe}_{0.88}\left[\mathrm{Si}_{0.12-x} \mathrm{Al}_{x}\right)_{13}\right.$ the balance between changes in the field dependence of the heat capacity with respect to overall $\Delta T_{\text {ad }}$ gain is highlighted, showing that a small amount of Al doping clearly offers some advantage for application.
\end{abstract}

DOI: 10.1103/PhysRevB.83.144415

\section{INTRODUCTION}

The magnetocaloric effect has received a growth in interest due to its application in environmentally friendly and energy efficient solid state magnetic cooling at room temperature. The current difficulty lies in finding a magnetic refrigerant with large entropy changes that could be implemented in a cooling process. Magnetocaloric materials that show giant changes of entropy (GMCE) because of the underlying first order nature of the transition (where first order character is identified by the observation of latent heat and a magnetic transition that is sharp in field and temperature) are of interest for application, but the associated hysteresis adds loss to the cooling cycle. ${ }^{1,2}$ As well as the balance between entropy change and hysteresis, it is also well established that the adiabatic temperature change, $\Delta T_{\text {ad }}$, is limited by the absolute magnitude of the heat capacity, and its changes in field. ${ }^{3}$ The first order magnetocaloric paramagnetic to ferromagnetic transition in the $\mathrm{La}\left(\mathrm{Fe}_{y} \mathrm{Si}_{1-y}\right)_{13}$ system appears to offer a unique combination of characteristics with large entropy change, $\Delta S$, of up to $30 \mathrm{JK}^{-1} \mathrm{~kg}^{-1}$ in field changes of 0-5 T (Ref. 4), coupled with remarkably small hysteresis, $\Delta H$, and a $T_{c}$ easily tunable by the interstitial addition of $\mathrm{H}$ (Ref. 4), or by doping with Co (Ref. 5).

The magnetovolume transition in $\mathrm{La}\left(\mathrm{Fe}_{y} \mathrm{Si}_{1-y}\right)_{13}$ is isotropic with respect to the crystal axes ${ }^{6,7}$ with an expansion, $\Delta V / V$, of the order of $1.2 \%$ for $y=0.88$ (Ref. 8), and is highly sensitive to pressure [where it has been shown that $d l n T_{c} / d P=-0.46 \mathrm{GPa}^{-1}$ for $\left.\mathrm{La}\left(\mathrm{Fe}_{0.89} \mathrm{Si}_{0.11}\right)_{13}\right]$ (Refs. 9 and 10). Chemical pressure by interstitial doping of $\mathrm{H}$ or $\mathrm{C}$ significantly increases the $T_{c}$ of a given material system ${ }^{11}$ but with varying impact on the magnetic entropy change. Substitutions of other rare earth elements such as $\mathrm{Pr}, \mathrm{Ce}, \mathrm{Nd}$, and $\mathrm{Gd}$ for La have been shown to increase $\Delta S$, but are also accompanied by a reduction in $T_{c}$ (Ref. 10). Co substitution for Fe can increase $T_{c}$, but is accompanied by a reduction in the entropy change as the phase transition moves from first order to second order. ${ }^{10}$

Phenomenological aspects of these observations can be understood within the framework of the Bean and Rodbell model $^{12}$ as a volume effect. This model, although simple, describes the key relation between the volume change
PACS number(s): 75.30.Sg, 75.30.Kz, 65.40.Ba

and $T_{c}$ :

$$
T_{c}=T_{0}\left(1+\beta \frac{\left(V-V_{0}\right)}{V_{0}}\right),
$$

where $T_{0}$ is the Curie temperature if the lattice is incompressible, $V$ is the volume, $V_{0}$ is the volume in the absence of the exchange interaction, and $\beta=d T_{c} / d V$. The origin of first order behavior is often cited as due to associated volume changes, and occurs when

$$
P \kappa \beta>1-\eta,
$$

where $P$ is the pressure, $\kappa$ the isothermal compressibility, and $\eta$ is defined as

$$
\frac{5}{2} \frac{[4 j(j+1)]^{2}}{(2 j+1)^{4}-1} N k_{B} \kappa T_{0} \beta^{2},
$$

where $j$ is the angular momentum of the magnetic ions.

The Bean Rodbell model is included here to provide a basic starting point for establishing the relationship between volume change and the order of the phase transition. Although a first principles electronic structure calculation has been performed in this system (see Ref. 13), the inclusion or extension of this work here would be outside the scope of this experimental paper.

For example, the interstitial addition of $\mathrm{H}$ (increased volume $V$ ) has been shown to increase $T_{c}$ while maintaining relatively large values of $\Delta S$. This was suggested recently to be due to the increase in volume related to the additional $\mathrm{H}$ atoms in the otherwise unoccupied $24 d$ site, coupled with a limited impact on the density of states. ${ }^{14}$ Similarly, substitution of $\mathrm{La}$ by other rare earths such as $\mathrm{Ce}, \mathrm{Pr}$, or $\mathrm{Nd}$ results in a decrease of $T_{c}$ (the ionic size of the heavier rare earth is smaller due to lanthanide contraction) as well as enhancing first order behavior by increased magnetic coupling between the rare earth and $\mathrm{Fe}$ ions. ${ }^{15}$ Although several papers have addressed the influence of doping directly onto atomic sites and interstitially, the impact of these substitutions on $\Delta T_{\text {ad }}$ has not been directly addressed.

Several extremely thorough studies by Fujita et al. supported the itinerant theory of metamagnetism in this material 
system. ${ }^{4,6,7,9,14,16}$ Indeed the Rhodes Wolfarth plot $^{17}$ suggests some itinerancy as well as the influence of local exchange due to the rare earth ion. ${ }^{18}$ Within the itinerant magnetism model the impact of spin fluctuations on the heat capacity is expected to be small compared to electronic contributions at temperatures far away from $T_{c}$ (Ref. 19), but at the critical point they can, in principle, dramatically enhance the heat capacity, ${ }^{19}$ as was suggested recently in the study of melt spun ribbons of $\mathrm{LaFe}_{11.4} \mathrm{Si}_{1.6}$ (Ref. 20).

The substitution of $\mathrm{Al}$ for $\mathrm{Si}$ encourages the formation of an antiferromagnetic (AFM) ground state, ${ }^{21}$ which is accompanied by a decrease in $T_{c}$ and a linear increase in the lattice parameters. This is counterintuitive to the simple volume effect model, where an increase in volume increases the $T_{c}$ because the partial substitution of $\mathrm{Al}$ for $\mathrm{Si}$ removes one electron from the valence band, and could also have a significant impact on the local variation of $\mathrm{Fe}^{\mathrm{II}}-\mathrm{Fe}^{\mathrm{II}}$ distances in the $\mathrm{NaZn}_{13}$ type structure ${ }^{22}$ introducing an element of disorder.

It was shown recently that for $x>0.09$ the AFM state will persist at low temperatures and a field induced magnetic history can be invoked due to the metastability of the ferromagnetic (FM) and AFM states. ${ }^{21}$ This field history was used to probe the low temperature heat capacity $\left(C=\gamma T+\beta T^{3}\right)$ to extract the electronic specific heat coefficient, $\gamma$ where $C_{E}=\gamma T$ and $\beta T^{3}$ is the lattice vibration term. ${ }^{23}$ For $x=0, \gamma=$ $9.5 \mathrm{~mJ} / \mathrm{g}$-atom $K^{2}$ in the FM state. For Al substituted samples in the AFM state $\gamma=11.7 \mathrm{~mJ} / \mathrm{g}$-atom $K^{2}$. For $x>0.093$ at low temperatures the material will be antiferromagnetic if prepared by zero field cooling (ZFC), but can be driven to the ferromagnetic state on the application of a magnetic field. This is defined as the field induced FM state (FIFM) as the sample is thereafter trapped in the FM state unless subject to a thermal cycle above the Curie temperature $T_{c}$. The FIFM state in $x=$ 0.093 exhibited a larger value of $\gamma=16.2 \mathrm{~mJ} / \mathrm{g}$-atom $K^{2}$, but this last value is heavily dependent on the temperature and field history. ${ }^{24}$ The increase in $\gamma$ for the high Al content suggests that there will be a larger contribution to the electronic heat capacity.

In this paper we show using a combination of magnetization and calorimetry data on $\mathrm{La}\left(\mathrm{Fe}_{0.88} \mathrm{Si}_{0.12-x} \mathrm{Al}_{x}\right)_{13}$ the impact of Al substitution on the entropy change $\Delta S$, and adiabatic temperature change $\Delta T_{\text {ad }}$. In the parent compound $(x=0)$ we demonstrate an unusually large field dependence of the heat capacity and show how it contributes significantly to the total $\Delta S$. Al substitution diminishes the large observed $\Delta C_{p}$, and also disrupts the first order phase transition resulting in smaller $\Delta S$. This reduction in $\Delta S$ is offset, however, by the relative gain in $\Delta T_{\mathrm{ad}}$ demonstrating that a small but finite $x$ is an attractive doping strategy as a route forward for application.

\section{EXPERIMENTAL METHOD}

The $\mathrm{La}\left(\mathrm{Fe}_{0.88} \mathrm{Si}_{0.12-x} \mathrm{Al}_{x}\right)_{13}$ samples were prepared by arc melting the constituents in an Ar atmosphere, which were then annealed in a quartz tube at $1323 \mathrm{~K}$ for 12 days, followed by quenching in room temperature water. A single phase $\mathrm{NaZn}_{13}$ type structure was confirmed by X-ray Diffraction (XRD). ${ }^{25,26}$ $\mathrm{X}$-Ray powder diffraction analysis was performed by fitting to the $\mathrm{LaFe}_{13}$ phase and $\alpha$-Fe phase (using the Powercell program). It was found that the $\alpha$-Fe contribution is small, less than $8 \%$ for all samples, and does not vary in any systematic way with $\mathrm{Al}$ doping. No other second phase contribution was found within error. The magnetic phase diagram of this system as a function of Al substitution is given in Ref. 24.

Magnetization measurements on approximately 10-20 mg semispherical ingots, hereafter referred to as "bulk" ingots, were carried out in an Oxford instruments 8T VSM capable of temperature 4-295 $\mathrm{K}$ at a field sweep rate of $0.5 \mathrm{~T} / \mathrm{min}$. Zero field (relaxation type) heat capacity measurements were carried out on the same bulk samples with the magnetic field in the same orientation using a quantum design Physical Properties Measurement System (PPMS) and on small microgram fragments using a $\mathrm{SiNi}$ microcalorimeter. The latter has been developed to measure the latent heat (adiabatic temperature probe) and heat capacity (ac microcalorimetry) separately, and with high precision as detailed elsewhere. ${ }^{20,27}$ Note that there can be significant error in the absolute value of the heat capacity in microcalorimetry due to the correct subtraction of the addenda. The bulk heat capacity measurements allow us to determine the absolute value of heat capacity away from the transition and overcome this problem. The adiabatic temperature probe relies on a correlation length of the first order phase transition of the order of $100 \mu \mathrm{m}$, which is the size of the fragment measured. The ac microcalorimetry mode avoids latent heat contamination due to the associated hysteresis of the first order phase transition. ${ }^{20}$ In this case, the magnitude of the ac temperature modulation was of the order of $0.07 \mathrm{~K}$, much smaller than the thermal hysteresis close to $T_{c}$ of approximately $0.3 \mathrm{~K}$ (Ref. 20 ).

It is known that in this system there is coincident isostructural volume expansion during the transition from the paramagnetic (PM) to the ferromagnetic (FM) state of the order of $1.2 \%$. Here we use the heater resistance, $R_{h}$, embedded in the silicon nitride membrane, as a measure of the expansion in two dimensions. ${ }^{28}$ The change of $R_{h}$ due to lattice expansion of the sample is not calibrated precisely, but it provides a useful and reproducible guide to volume changes alongside heat capacity measurements.

\section{RESULTS AND DISCUSSION}

The isothermal $M-H$ loops of samples with $x=0,0.048$, and 0.081 are shown in Fig. 1. Notice the significant hysteresis in Fig. 1(a), which has contributions due to local heating ${ }^{29}$ and pressure $^{30,31}$ effects as well as the intrinsic hysteresis in the system. The intrinsic hysteresis, as defined in Ref. 29 is 0.05 $\mathrm{T}$ close to $T_{c}$. As the temperature is increased and the critical field $H_{c}$ increases, the associated hysteresis diminishes. After Al substitution the hysteresis disappears. We attribute this to a crossover to a second order phase transition, which is consistent with an absence of latent heat in all the finite $x$ samples.

Further substitution of $\mathrm{Al}$ for Si results in a stable AFM state in low fields, as indicated by the sharp low field metamagnetic transition for $x=0.081$ [see Fig. 1(c)], similar to the trends seen in studies of $\mathrm{La}(\mathrm{Fe}, \mathrm{Al})_{13}$ (Ref. 32). By applying a field of $1 \mathrm{~T}$ the AFM state is quenched, as indicated by the smooth $M(T)$ curves shown in Fig. 2. Notice that the impact of $\mathrm{Al}$ substitution on the $1 \mathrm{~T}$ field cooled $M(T)$ curve is a gradual broadening, consistent with either a loss of first order behavior, or disorder broadening of the first order phase transition. In 


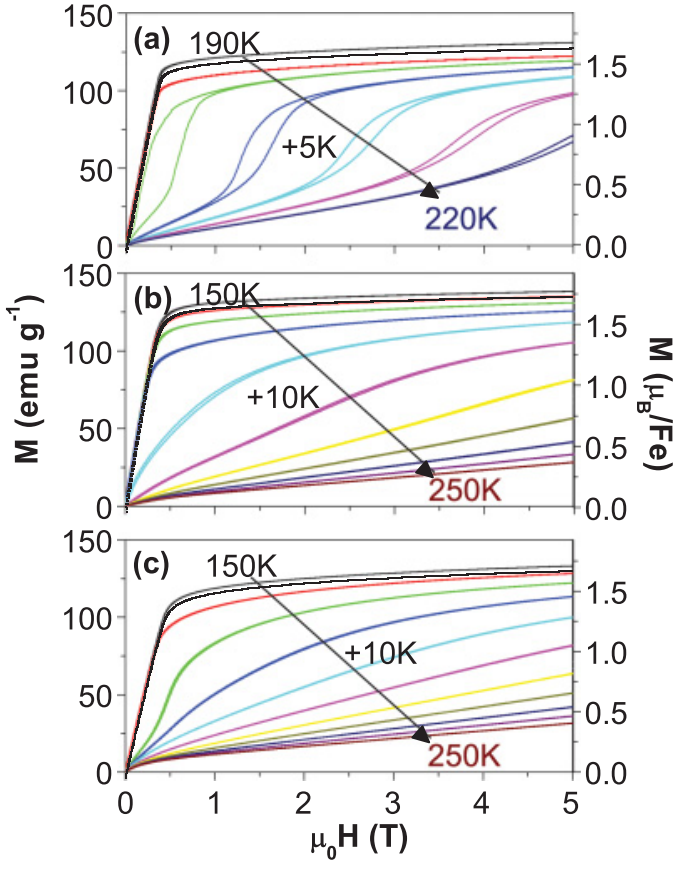

FIG. 1. (Color online) Magnetic behavior of $\mathrm{La}\left(\mathrm{Fe}_{0.88} \mathrm{Si}_{0.12-x}\right.$ $\left.\mathrm{Al}_{x}\right)_{13}$ as $x$ is increased. $M-H$ loops for (a) $x=0$, (b) $x=0.048$, and (c) $x=0.081$ for a field sweep rate of $0.5 \mathrm{~T} / \mathrm{min}$.

fields of $0.1 \mathrm{~T}$ the AFM state persists in $x=0.081$, as indicated by the feature highlighted by the arrow in Fig. 2. A schematic phase diagram can be constructed from magnetization and heat capacity data, with $H_{c}$ determined as the point at which the maxima in $C(H)$ or $d M / d H$ occur, as shown in Fig. 3.

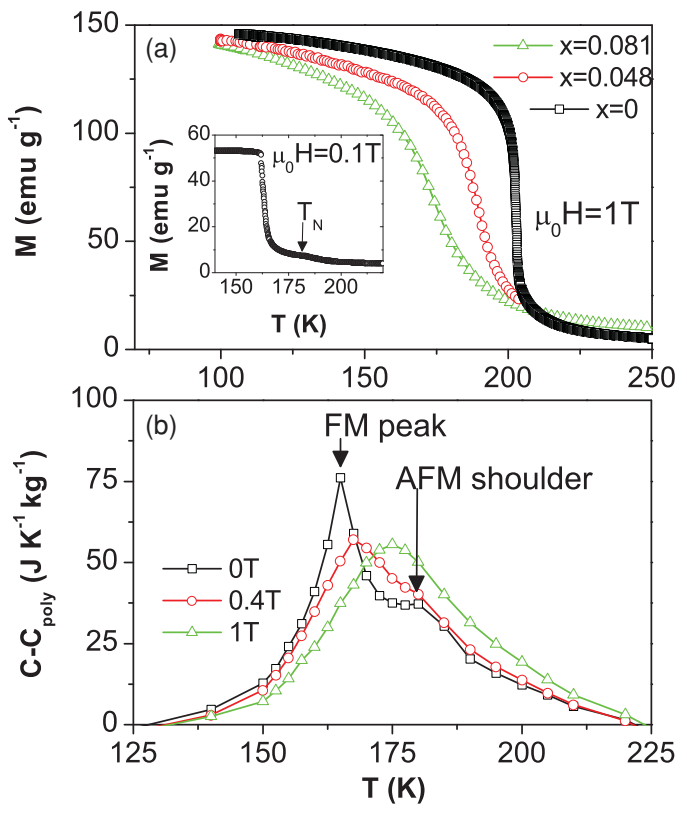

FIG. 2. (Color online) Magnetic and calorimetric behavior of $\mathrm{La}\left(\mathrm{Fe}_{0.88} \mathrm{Si}_{0.12-x} \mathrm{Al}_{x}\right)_{13}$. (a) $M(T)$ curves in $1 \mathrm{~T}$ and inset $0.1 \mathrm{~T}$ for $x=0.081$. (b) Peak and shoulder feature observed in heat capacity measurements of sample $x=0.081$, highlighted by removing the polynomial background heat capacity.

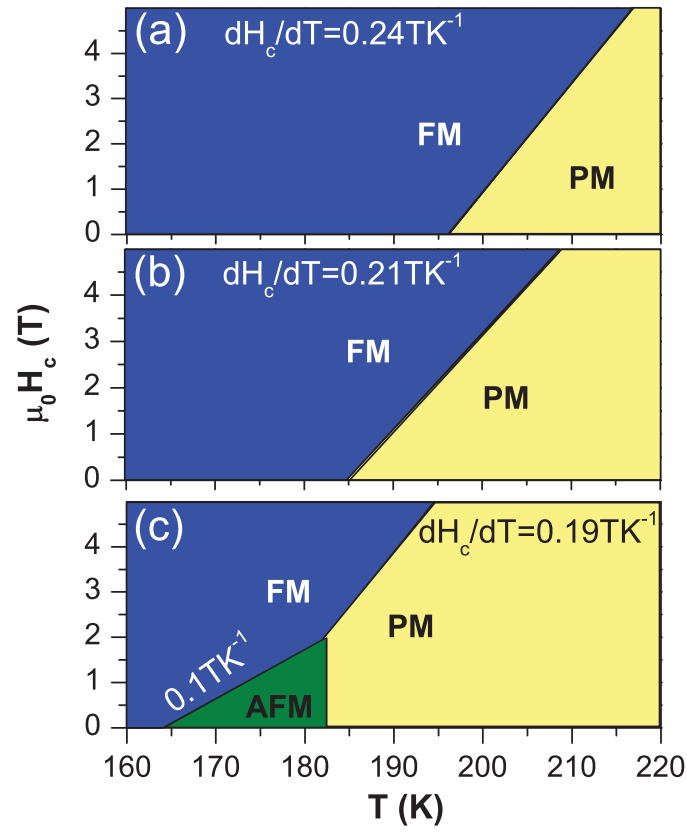

FIG. 3. (Color online) $H-T$ phase diagrams and field dependent heat capacity close to $T_{c}$, constructed from magnetization and heat capacity data for (a) $x=0$, (b) $x=0.048$, and (c) $x=0.081$. The AFM state was determined from the position of the shoulder feature in the heat capacity data and the bump observed in $0.1 \mathrm{~T}$ field cooled $M-T$ curves shown in Fig. 2.

The zero field heat capacity of bulk ingots as a function of temperature are shown for all three compounds in the inset to Fig. 4(a). The purpose was to measure the absolute heat capacity away from the phase transition and relatively coarse temperature steps were taken (such that the absolute heat capacity at the transition will be poorly resolved and not accurate). The measurement is useful to compare the absolute heat capacity value for all the samples away from the transition temperature (and as the inset shows, the heat capacity is very similar for all samples in this regime). In contrast, the microcalorimetry method allows the ac heat capacity changes shown in Fig. 4(a) and absolute latent heat magnitude to be measured independently and with high precision. ${ }^{20}$ Latent heat was observed for sample $x=0$ only, as shown in Fig. 4(b).

Figure 5 shows the change in heat capacity, $\Delta C\left(=C_{\mathrm{H}^{-}} C_{0 T}\right)$ as determined by the ac microcalorimetry probe, as a function of applied magnetic field for each sample in the vicinity of $T_{c}$. The gradual broadening of the phase transition as $x$ increases results in a decrease of the gradient $d H_{c} / d T$. To put the heat capacity changes observed here into context, the benchmark material for working prototype fridges, $\mathrm{Gd}$, exhibits a change in heat capacity due to field of the order of $50 \mathrm{JK}^{-1} \mathrm{~kg}^{-1}$, which is a similar magnitude as the $x=0.081$ sample shown in Fig. 5(c). The $x=0$ sample exhibits changes that are 20 times larger.

The magnetization curve captures all the field driven entropy change in the system. The latent heat observed in the $x=0$ sample [Fig. 4(b)] contributed approximately $30 \%$ of the total entropy change, $\Delta S_{\text {tot }}$. A direct comparison of $\Delta S_{\mathrm{HC}}$ to $\Delta S_{\mathrm{Max}}$ for the $x=0.048$ and 0.081 samples suggests 

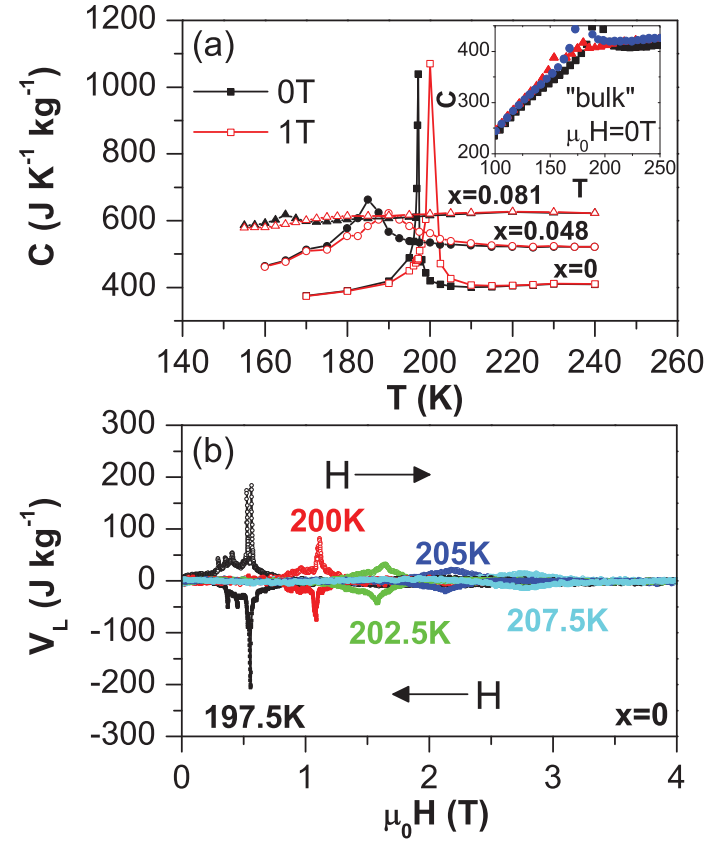

FIG. 4. (Color online) (a) Heat capacity as a function of temperature taken using microcalorimetry. The $x=0.048$ and 0.081 have been offset by 100 and $200 \mathrm{~J} K^{-1} \mathrm{~kg}^{-1}$, respectively. Note that temperature steps of $0.5 \mathrm{~K}$ were required to capture the full heat capacity peak in $0 \mathrm{~T}$. The inset shows $C(T)$ for all three bulk samples measured in the PPMS where the symbols used refer to the same samples as the main figure. (b) Latent heat observed for sample $x=0$. Arrows indicate field direction where for field increase a temperature increase is observed.

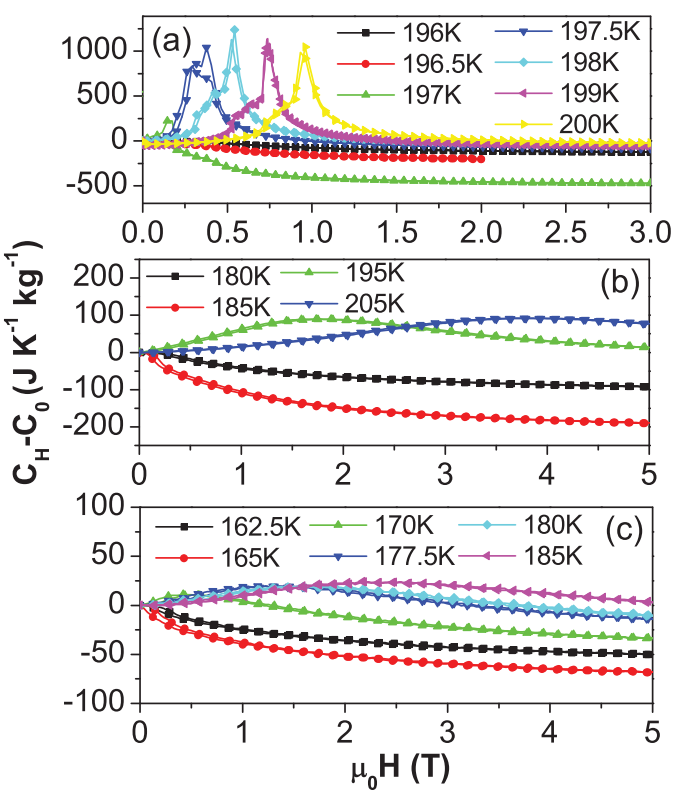

FIG. 5. (Color online) Change in heat capacity, $C_{H}-C_{0 T}$, as a function of field for (a) $x=0$ (note the very large changes in heat capacity as seen elsewhere, Refs. 20, 24), (b) $x=0.048$, and (c) $x=$ 0.081 .

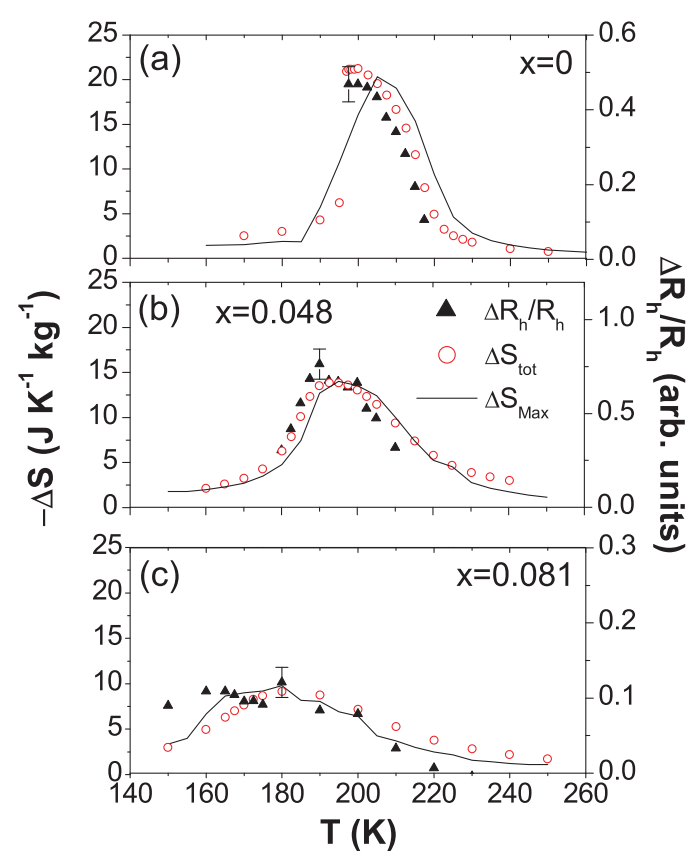

FIG. 6. (Color online) Entropy change determined from microcalorimetry, $\Delta S_{\mathrm{tot}}$, compared to that calculated from the Maxwell relation applied to isothermal $M-H$ loops, $\Delta S_{\text {Max }}$. The latent heat observed in $x=0$ contributed to approximately $30 \%$ of the total entropy change, $\Delta S_{\text {tot }}$. Magnetostriction measured as a change in the heater membrane resistance, $\Delta R_{h} / R_{h}$, is shown on the right-hand axis. The error bar in $\Delta R_{h} / R_{h}$ is indicative of the error at all temperatures. The field change is $0-5 \mathrm{~T}$.

that the latent heat contribution must either be very small or absent, indeed we cannot detect it by direct measurement. As the first order transition is governed by magnetovolume coupling, the question arises as to whether the magnetic and volume changes are completely decoupled in the finite $x$ samples.

The measured change in heater resistance, $\Delta R_{h} / R_{h}$, can be used as an indication of the volume change for a given sample as a function of temperature. ${ }^{28}$ In Fig. 6 it is plotted alongside the entropy changes for all samples studied. Notice that for $x=$ 0 there is good agreement between the temperature variation of $\Delta R_{h} / R_{h}$ and the total entropy change. As $x$ increases, $\Delta R_{h} / R_{h}$ broadens consistent with reports elsewhere ${ }^{15}$ and becomes less strongly coincident with $\Delta S$, suggesting decoupling of the magnetic and volume transitions. The magnetovolume coupling can be described in terms of

$$
\frac{\Delta V}{V}=\kappa C M(T)^{2}
$$

where $\Delta V / V$ is the volume change, $\kappa$ is the compressibility, and $C$ is the magnetovolume coupling constant. ${ }^{8}$ A direct comparison to the data in Fig. 6 suggests that as $x$ increases the magnetovolume coupling constant $C$ decreases (assuming that there is no change in $\kappa$ ). Studies by Irisawa et al. also showed a reduced volume change in $\mathrm{La}\left(\mathrm{Fe}_{0.88} \mathrm{Al}_{0.12}\right)_{13}$ of $1 \%$ compared to that measured in $\mathrm{La}\left(\mathrm{Fe}_{0.88} \mathrm{Si}_{0.12}\right)_{13}$ of $1.2 \%$. Interestingly there is still finite coupling in the systems that 
show second order behavior. This suggests from Eq. (2) that there is a change in $\kappa$ or $d T_{c} / d V$.

The Bean Rodbell model was proposed to explain the occurrence of first order behavior in a FM-PM phase transition. It requires a volume change to introduce some energy barrier that needs to be overcome for the transition to occur. The increase of $\Delta R_{h} / R_{h}$ and observation of latent heat is a direct consequence of the larger volume change involved in the $x=0$ sample. By substituting Al for $\mathrm{Si}$, the simple volume effect, which we might expect to increase $T_{c}$, is in competition with a change in the electronic density of states (indicated by the change of absolute heat capacity) that reduces the volume change at the PM-FM phase transition and decreases $T_{c}$. Spin fluctuations also reduce the effective energy barrier (renormalize the energy scale) associated with the volume change, $E_{b}$, and for $x=$ 0.048 and 0.081 , the spin fluctuations are able to renormalize the energy scale such that $E_{b}$ is effectively zero. For $x=0$ a finite barrier exists, the system is clearly first order, and there is a huge increase in the heat capacity very close to the phase transition.

The large changes of heat capacity in a field directly impact the achievable adiabatic temperature change, $\Delta T_{\mathrm{ad}}$, which can be calculated from the measured heat capacity and magnetization using Eq. (4) $)^{33}$

$$
\Delta T_{\mathrm{ad}}=-\mu_{0} \int_{H 1}^{H 2}\left(\frac{T}{c_{p}(H, T)} \times \frac{\partial M(T, H)}{\partial T}\right)_{H} d H .
$$

Here we take the isothermal bulk magnetization measurements shown in Fig. 1 and the heat capacity determined from microcalorimetry as a function of field (Fig. 5) to perform numerical summation as described in Eq. (5). If the change in heat capacity across the phase transition can be taken to be field independent, then the determination of $\Delta T_{\text {ad }}$ is much simpler, as described by Eq. (6). Often, where field-dependant heat capacity data are not available, Eq. (6) is implemented to determine $\Delta T_{\mathrm{ad}}$ indirectly, which was shown previously to be invalid. ${ }^{33}$

$$
\begin{aligned}
& \Delta T_{\mathrm{ad}}(T, H) \\
& =-\mu_{0} \sum_{H_{i}=0,+\Delta H}^{H}\left(\frac{T}{c_{p}(H, T)}\right. \\
& \left.\times \frac{M\left(T, H_{i}\right)-M\left(T+\Delta T, H_{i}\right)}{\Delta T}\right) \Delta H, \\
& \Delta T_{\mathrm{ad}}(T) \approx-\frac{T}{c_{p}(T)} \Delta S(T) .
\end{aligned}
$$

The results of these calculations are shown in Fig. 7. For $x=0$ the immediate gain from the first order phase transition is the large entropy change, but this is tempered by the reduction in $\Delta T_{\text {ad }}$ due to the large field dependent changes in heat capacity. Note that for local moment systems such as MnAs, $\mathrm{Gd}_{5} \mathrm{Ge}_{2} \mathrm{Si}_{2}$, and CoMnSi (Ref. 34), a larger proportion of the entropy change is contained in the latent heat term and the heat capacity changes are negligible. This is an important point. The reduction in $\Delta T_{\text {ad }}$ we see here in $\mathrm{La}\left(\mathrm{Fe}_{0.88} \mathrm{Si}_{0.12-x} \mathrm{Al}_{x}\right)_{13}$ is a feature of the itinerant metamagnetic phase transition and
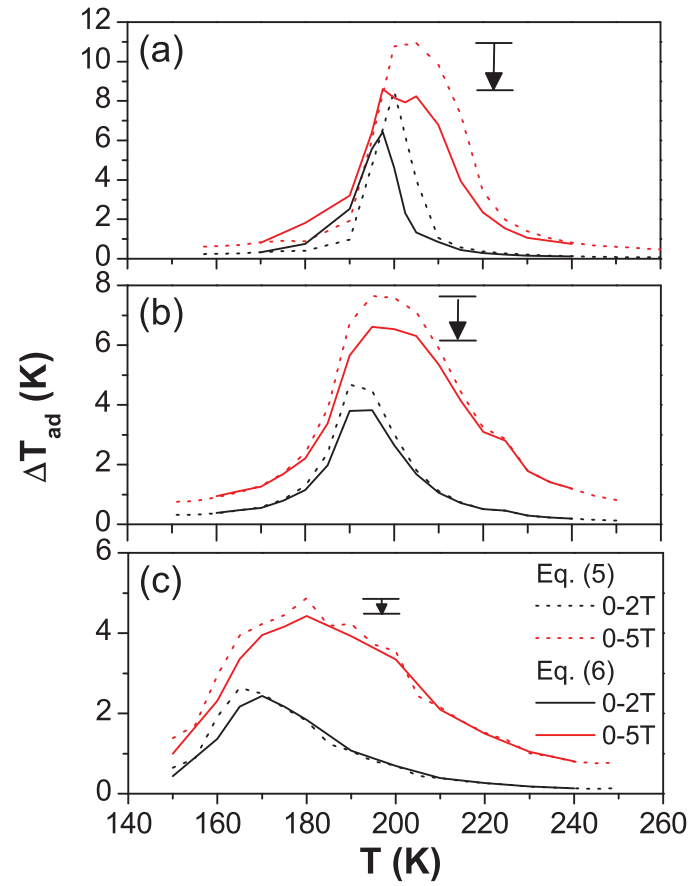

FIG. 7. (Color online) The adiabatic temperature change, $\Delta T_{\text {ad }}$, determined separately from calorimetry and bulk magnetization data [using Eq. (5)] compared to that estimated from the baseline heat capacity of approximately $400 \mathrm{JK}^{-1} \mathrm{~kg}^{-1}$ determined from Fig. 4 [using Eq. (6)]. The arrows indicate the difference between calculated values of the maximum $\Delta T_{\text {ad }}$ using Eqs. (5) and (6).

huge associated heat capacity changes, not of first order phase transitions in general.

As the $\mathrm{Al}$ content is increased the large changes in heat capacity associated with spin fluctuations decrease, resulting in a decrease of the "relative loss" of $\Delta T_{\text {ad }}$, although the lower transition temperatures of $x=0.048$ and $x=0.081$ also reduce $\Delta T_{\mathrm{ad}}$ further [see Eq. (6)]. Hence for optimum performance there is a competition between different effects which influence the final $\Delta T_{\text {ad }}$ that can be achieved.

\section{CONCLUSION}

To summarize, we have made an in depth study by microcalorimetry of the $\mathrm{La}\left(\mathrm{Fe}_{0.88} \mathrm{Si}_{0.12-x} \mathrm{Al}_{x}\right){ }_{13}$ system. We have shown that partial substitution of $\mathrm{Al}$ on the $\mathrm{Si}$ site of $\mathrm{La}\left(\mathrm{Fe}_{0.88} \mathrm{Si}_{0.12}\right)_{13}$ gradually broadens the magnetic transition resulting in a decrease of $\Delta S$. By direct measurement of the heat capacity term (and its separation from the latent heat contribution) we have been able to capture the magnitude of the spin fluctuation contribution to the heat capacity in the vicinity of the phase transition, as the phase transition temperature is tuned in the applied magnetic field. The spin fluctuation term has a nonmonotonic dependence on field and temperature, first increasing as temperature is increased away from $T_{c}$, and then broadening as the fluctuations are suppressed in the applied magnetic field. Substitution by $\mathrm{Al}$ results in a broadening of the volume change and an increasing lack of registration between volume and magnetic changes suggesting decoupling between the two. When the $\Delta T_{\mathrm{ad}}$ is calculated indirectly from field-dependent heat capacity and 
magnetization data, a significant reduction in the maximum $\Delta T_{\text {ad }}$ compared to the ideal first order transition is observed due to the strong field dependence of the heat capacity. Some systems show "ideal first order behavior", for example our previous report on CoMnSi (Ref. 34). Such systems are however accompanied by large hysteresis, which is detrimental to the refrigeration cycle efficiency. For low concentrations (less than we have studied here), Al-substituted materials may offer advantages for application, limiting the impact of the field change of heat capacity to the maximal $\Delta T_{\text {ad }}$ and yet allowing the entropy change to remain large. For these materials, the addition of interstitial $\mathrm{H}$ could then be used to tune the $T_{c}$ value into the application-relevant temperature range.

\section{ACKNOWLEDGMENTS}

This work was supported by EPSRC Platform Grant No. EP/E016243/1 — a platform to develop and utilize characterization tools for functional magnetic materials.
${ }^{1}$ V. K. Pecharsky and K. A. Gschneidner Jr., Phys. Rev. Lett. 78, 004494 (1997).

${ }^{2}$ A. de Campos, D. L. Rocco, A. M. G. Carvalho, L. Caron, A. A. Coelho, S. Gama, L. M. da Silva, F. C. G. Gandra, A. O. dos Santos, L. P. Cardoso, P. J. von Ranke, and N. A. de Oliveira, Nat. Mater. Lett. 5, 802 (2006).

${ }^{3}$ K. A. Gschneidner Jr., V. K. Pecharsky, and A. O. Tsokol, Rep. Prog. Phys. 68, 1479 (2005).

${ }^{4}$ A. Fujita, S. Fujieda, Y. Hasegawa, and K. Fukamichi, Phys. Rev. B 67, 104416 (2003)

${ }^{5}$ F. X. Hu, J. Gao, X. L. Qian, M. Ilyn, A. M. Tishin, J. R. Sun, and B. G. Shen, J. Appl. Phys. 97, $10 \mathrm{M} 303$ (2005).

${ }^{6}$ A. Fujita, Y. Akamatsu, and K. Fukamichi, J. Appl. Phys. 85, 4756 (1999).

${ }^{7}$ A. Fujita, K. Fukamichi, K. Koyama, and K. Watanabe, J. Appl. Phys. 95, 6687 (2004).

${ }^{8}$ K. Fukamichi and A. Fujita, J. Mater. Sci. Technol. 16, 167 (2000).

${ }^{9}$ A. Fujita, K. Fukamichi, M. Yamada, and T. Goto, Phys. Rev. B 73, 104420 (2006).

${ }^{10}$ J. Lyubina, K. Nenkov, L. Schultz, and O. Gutfleisch, Phys. Rev. Lett. 101, 177203 (2008).

${ }^{11}$ B. G. Shen, J. R. Sun, F. X. Hu, H. W. Zhang, and Z. H. Cheng, Adv. Mater. 21, 4545 (2009).

${ }^{12}$ C. P. Bean and D. S. Rodbell, Phys. Rev. 126, 104 (1962).

${ }^{13}$ M. D. Ku'zmin and M. Richter, Phys. Rev. B 76, 092401 (2007).

${ }^{14}$ A. Fujita, S. Fujieda, and K. Fukamichi, J. Magn. Magn. Mater. 321, 3553 (2009).

${ }^{15}$ L. Jia, J. R. Sun, J. Shen, Q. Y. Dong, F. X. Hu, T. Y. Zhao, and B. G. Shen, Appl. Phys. Lett. 92, 182503 (2008).

${ }^{16}$ K. Irisawa, A. Fujita, K. Fukamichi, M. Yamada, H. Mitamura, T. Goto, and K. Koyama, Phys. Rev. B 70, 214405 (2004).

${ }^{17}$ P. Rhodes and E. P. Wohlfarth, Proc. R. Soc. London A 273, 247 (1963).
${ }^{18}$ J. D. Zou, B. G. Shen, and J. R. Sun, J. Phys. Condens. Matter 19, 196220 (2007).

${ }^{19}$ M. Shimizu, Rep. Prog. Phys. 44, 329 (1981).

${ }^{20}$ K. Morrison, J. Lyubina, J. D. Moore, A. D. Caplin, K. G. Sandeman, O. Gutfleisch, and L. F. Cohen, J. Phys. D 43, 132001 (2010).

${ }^{21}$ X. B. Liu, Z. Altounian, and A. D. Beath, J. Appl. Phys. 95, 6924 (2004).

${ }^{22}$ A. Fujita, S. Fujieda, and K. Fukamichi, J. Magn. Magn. Mater. 321, 3553 (2009).

${ }^{23}$ C. Kittel, Thermal Physics (John Wiley \& Sons, New York, 1969).

${ }^{24}$ S. M. Podgornykh and I. M. Chirkova, J. Phys. Conf. Ser. 200, 032057 (2010).

${ }^{25}$ S. M. Podgornykh and Ye. V. Shcherbakova, Phys. Rev. B 73, 184421 (2006).

${ }^{26} \mathrm{~S}$. M. Podgornykh et al., Physics of Metal and Metallography (to be published).

${ }^{27}$ Y. Miyoshi, K. Morrison, J. D. Moore, A. D. Caplin, and L. F. Cohen, Rev. Sci. Instrum. 79, 074901 (2008).

${ }^{28}$ A. A. Minakov, S. B. Roy, Y. V. Bugoslavsky, and L. F. Cohen, Rev. Sci. Instrum. 76, 043906 (2005).

${ }^{29}$ J. D. Moore, K. Morrison, K. G. Sandeman, M. Katter, and L. F. Cohen, Appl. Phys. Lett. 95, 252504 (2009).

${ }^{30}$ J. Lyubina, R. Schäfer, N. Martin, L. Schultz, and O. Gutfleisch, Adv. Mater. 33, 3735 (2010).

${ }^{31}$ K. Morrison, UoL Ph.D. Thesis, Imperial College London, UK, 2010.

${ }^{32}$ F. X. Hu, B. G. Shen, J. R. Sun, G. J. Wang, and J. Gao, J. Appl. Phys. 101, 09C525 (2007).

${ }^{33}$ V. K. Pecharsky and K. A. Gschneidner Jr., J. Appl. Phys. 86, 565 (1999).

${ }^{34}$ K. Morrison, A. Barcza, J. D. Moore, K. G. Sandeman, M. K. Chattopadhyay, S. B. Roy, A. D. Caplin, and L. F. Cohen, J. Phys. D: Appl. Phys. 43, 195001 (2010). 